\title{
EXIQPON
}

\section{Functional studies of microRNA based on knockdown using Locked Nucleic Acid probes}

\author{
Here we present a microRNA (miRNA) knockdown application, based on the use of Locked Nucleic Acid \\ $\left(\right.$ LNA $\left.^{\mathrm{TM}}\right)$-modified probes. The use of LNA for the knockdown of miRNA is particularly effective because \\ these probes have high affinity for their short RNA targets and excellent mismatch-discrimination \\ capability. Here we demonstrate that LNA-modified probes, termed miRCURY ${ }^{\mathrm{TM}}$ LNA Knockdown \\ probes, are highly specific and potent tools for functional analysis of miRNAs.
}

\section{The importance of microRNAs}

Mature miRNAs are 16-29 nucleotides (nt) long and are made by sequential processing from longer hairpin transcripts by the RNase III ribonucleases Drosha and Dicer. miRNAs have emerged as an important class of short endogenous RNAs that act as regulators of gene expression by base-pairing with their target mRNAs. This results in repression of translation of the target mRNAs or in their degradation, depending on the degree of sequence complementarity between the miRNA and its mRNA target.

The study of microRNAs has become of considerable interest to researchers who study the control of gene expression, or search for biomarkers and therapeutic targets. The explosion of interest in miRNAs in the past two years necessitates effective tools for functional analysis of these molecules. For this purpose, LNA-based miRNA knockdown has been demonstrated to be very efficient and reliable ${ }^{1-5}$.

\section{Locked Nucleic Acids}

LNAs are a class of nucleic acid analogs in which the ribose ring is 'locked' with a methylene bridge connecting the 2'-O atom with the 4'-C atom. LNA nucleosides containing the six common nucleobases (thymidine, cytosine, guanosine, adenosine, uridine and methyl cytosine) that appear in DNA and RNA are able to form base pairs with their complementary nucleosides according to standard Watson-Crick base-pairing rules.

The inclusion of LNAs in oligonucleotides dramatically increases the affinity for the target. The addition of a single LNA nucleotide typically increases the melting temperature $\left(T_{\mathrm{m}}\right)$ of the oligonucleotide -target duplex by $2-6{ }^{\circ} \mathrm{C}$, in sequences below $30 \mathrm{nt}$ in length. Furthermore, it has been demonstrated that LNA-containing probes have excellent mismatch discrimination capabilities, expressed as a

\section{Søren Rasmussen \& Peter Roberts}

Exiqon, Bygstubben 9, DK-2950 Vedbæk, Denmark. Correspondence should be addressed to P.R. (ptr@exiqon.com).

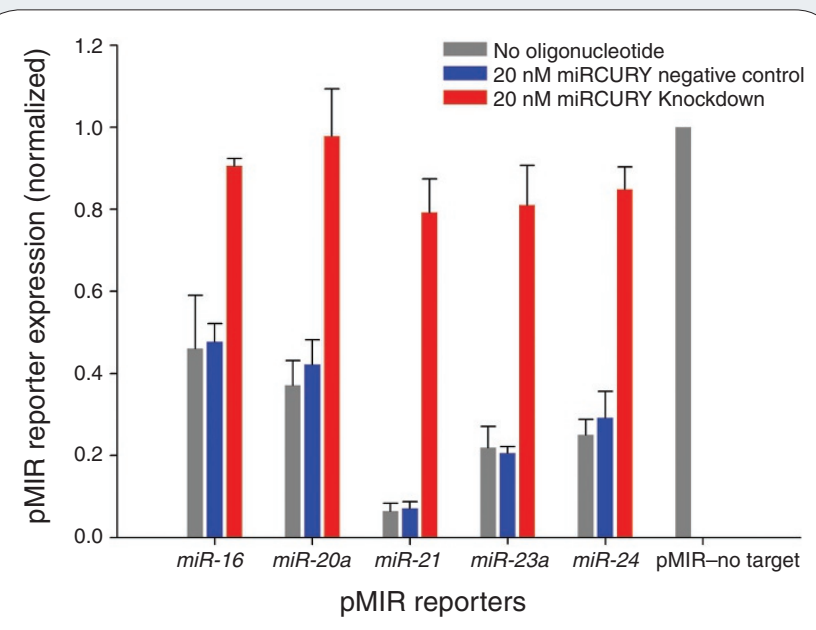

Figure $\mathbf{1}$ | Representative knockdown of microRNAs by miRCURY LNA Knockdown probes. Expression levels of the indicated miR reporter constructs carrying a miR complementary region. The no-target control is the parental vector without a miR target. HeLa cells were cotransfected with miR reporter (firefly luciferase), pGL4.13-Renilla (for normalization) and miRCURY LNA Knockdown probes against the named hsa-mir targets. Expression levels were measured using Dual-Glo ${ }^{\text {TM }}$ Luciferase (Promega). Each experiment was performed at least three times, in triplicate, and a representative example is shown.

substantial perturbation in $T_{\mathrm{m}}$ against a mismatched target ${ }^{6}$. These LNA-containing oligonucleotides have low cytotoxicity and are easy to transfect.

\section{Developing miRCURY LNA Knockdown probes}

In addition to being short, a fair proportion of miRNAs have high sequence similarity. For example, some miRNAs differ from each other by only a single base. The short length of miRNAs and the relatedness of their sequences are recurring themes in the miRNA world. In this context, the properties of LNAs discussed above make them excellent tools for studying miRNAs—LNA-based knockdown allows for highpotency knockdown without compromising specificity. 


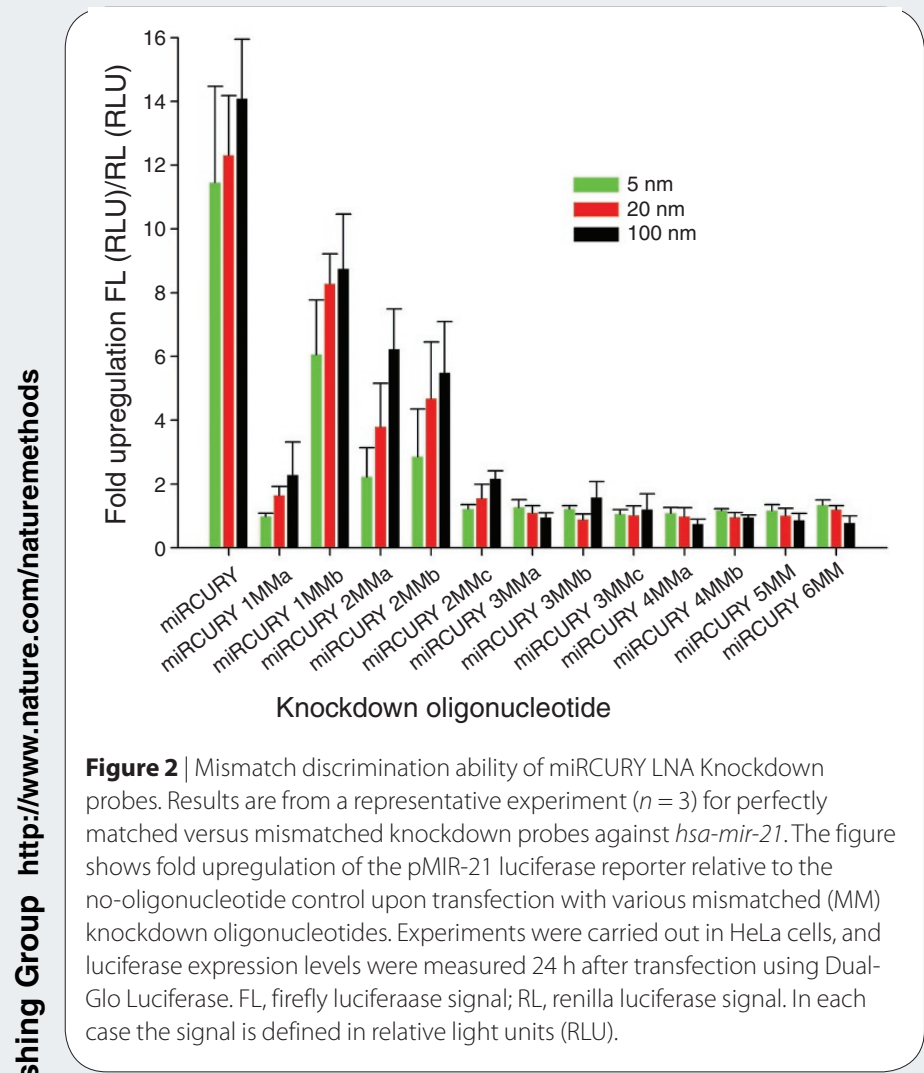

LNAs have proven to be an excellent tool in miRNA research, particularly in the areas of miRNA profiling and in situ hybridization ${ }^{7,8}$. Research into miRNA using LNA-based tools has generated important information concerning the roles of miRNAs as biomarkers, the evolution of miRNAs in vertebrates and their role in developmental biology. LNAs, however, can also be used for more functional studies of miRNAs. We and others have found that LNA-based miRNA knockdown is very efficient and reliable for this purpose.

Exiqon uses in-house bioinformatics expertise to optimally design probes-called miRCURY LNA Knockdown probes-that result in specific knockdown of miRNAs (Fig. 1).

LNA-based knockdown probes have high potency without compromising specificity (Fig. 2). A large decrease in knockdown inhibition is demonstrated with the introduction of a single mismatch between knockdown probe and miRNA target. The introduction of two mismatches between knockdown probe and miRNA target leads to complete elimination of inhibition.

miRCURY LNA Knockdown probes are also more effective at knocking down miRNAs than standard competing methods such as DNAbased probes and 2'-O-methyl (2'-OMe)-based probes (Fig. 3).

\section{Conclusion}

LNA-based oligonucleotide probes have enhanced studies of miRNA by providing improved detection of these short RNAs owing to enhanced binding properties. These properties have now been

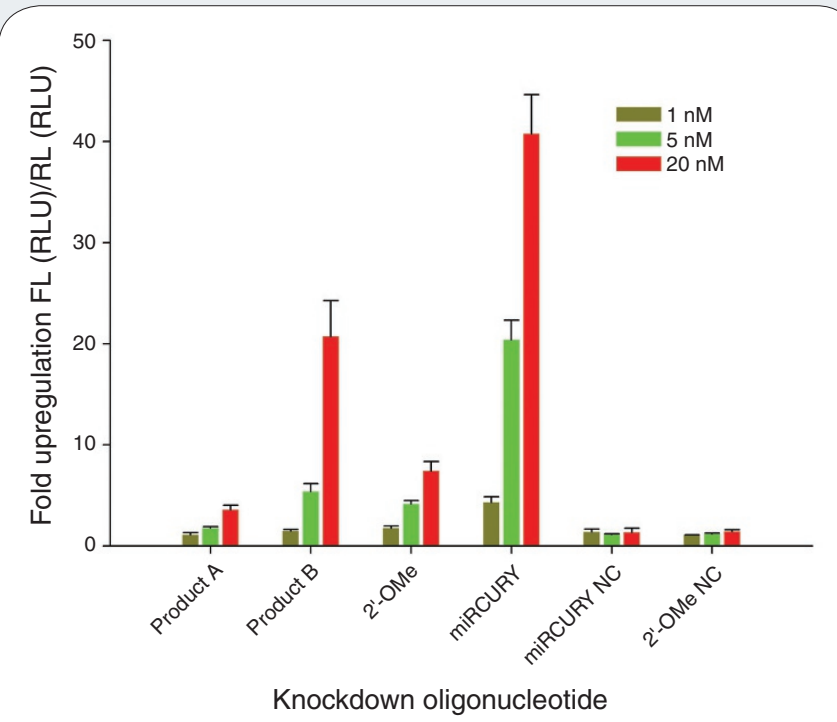

Figure 3 | Comparison of the effectiveness of different knockdown methods. The diagram shows results of a representative experiment $(n=3)$, indicating fold upregulation, relative to the untreated control of the pMIR-21 reporter (firefly luciferase) when cotransfected with various hsa-mir-21 knockdown products. Expression levels in MCF7 cells were measured $60 \mathrm{~h}$ after transfection. The negative control (NC) oligonucleotides are 2'-OMe or LNA-DNA mixmers with a random sequence showing no significant complementarity to any known miRNAs.

exploited to develop probes that can be used to effectively knock down miRNA function in cells. These miRCURY LNA Knockdown probes work by providing specific knockdown of miRNA with much higher potency than traditional antisense technologies. Several publications demonstrate the effectiveness of these probes ${ }^{1-4}$.

1. Fazi, F. et al. A minicircuitry comprised of microRNA-223 and transcription factors NFI-A and C/EBP $\alpha$ regulates human granulopoiesis. Cell 123, 819-831 (2005).

2. Orom, U.A., Kauppinen, S. \& Lund, A.H. LNA-modified oligonucleotides mediate specific inhibition of microRNA function. Gene 372, 137-141 (2006).

3. Naguibneva, l. et al. The microRNA miR-181 targets the homeobox protein Hox-A11 during mammalian myoblast differentiation. Nat. Cell Biol. 8, 278-284 (2006).

4. Boutz, P.L., Chawla, G., Stoilov, P. \& Black, D.L. MicroRNAs regulate the expression of the alternative splicing actor $\mathrm{nPTB}$ during muscle development. Genes Dev. 21, 71-84 (2007).

5. Triboulet, R. et al. Suppression of microRNA-silencing pathway by HIV1 during virus replication. Science, published online 22 February 2007 (10.1126/science.1136319).

6. You, Y. et al. Design of LNA probes that improve mismatch discrimination. Nucleic Acids Res. 34, e60 (2006).

7. Wienholds, E. et al. MicroRNA expression in zebrafish embryonic development. Science 309, 310-311 (2005).

8. Castoldi, M. et al. A sensitive array for microRNA expression profiling (miChip) based on Locked Nucleic Acids (LNA). RNA 12, 913-920 (2006).

9. Davis, S., Lollo, B., Freier, S. \& Esau, C. Improved targeting of miRNA with antisense oligonucleotides. Nucleic Acids Res. 34, 2294-2304 (2006).

This article was submitted to Nature Methods by a commercial organization and has not been peer reviewed. Nature Methods takes no responsibility for the accuracy or otherwise of the information provided. 